\title{
Convergence Study for Rock Unconfined Compression Test Using Discrete Element Method
}

\section{Muhammad Irfan Shahrin ${ }^{*}$, Rini Asnida Abdullah ${ }^{1}$, Mohd Nur Asmawisham Alel $^{1}$, Radzuan Saari ${ }^{1}$, Nurul Ain Ibrahim ${ }^{2}$, Nur Ain Mat Yusof ${ }^{3}$, Mohd Fadhli Abd Rashid ${ }^{1}$}

\author{
${ }^{1}$ School of Civil Engineering, Faculty of Engineering, \\ Universiti Teknologi Malaysia, 81310 Johor Bahru, Johor, MALAYSIA \\ ${ }^{2}$ Faculty of Engineering and Quantity Surveying, \\ INTI International University, 71800 Nilai, Negeri Sembilan, MALAYSIA \\ ${ }^{3}$ Faculty of Civil Engineering, \\ Universiti Teknologi MARA Pahang, 26400 Bandar Tun Abdul Razak, Jengka, Pahang, MALAYSIA \\ *Corresponding Author
}

DOI: https://doi.org/10.30880/ijie.2021.13.03.014

Received 20 December 2020; Accepted 01 May 2021; Available online 06 June 2021

\begin{abstract}
Mesh convergence is a vital issue that needs to be addressed in a numerical model. This study investigated the effects of mesh element number on the Discrete Element Method (DEM) to granite rock response under compression loading. This study used the 3D finite-element code LS-DYNA to model the Unconfined Compression Test (UCT) numerical simulation. Models with five different mesh types were conducted for convergence mesh, namely normal mesh, fine mesh, super fine mesh, coarse mesh, and super coarse mesh. The mesh convergence of rock media has been conducted using DEM and steel plates simulated using the Finite Element Method (FEM). The DEM-FEM numerical analysis is compared with the results obtained from the experimental test. The best mesh was obtained as the simulation could reproduce the stress-strain curve trends, the failure behaviour and compression strength observed in the experimental test. The normal mesh was selected as the best mesh type in this study based on the comparisons that have been made. This study shows that the DEM-FEM numerical simulation can represent granite rock and can be used for further study based on mesh convergence.
\end{abstract}

Keywords: Convergence, discrete element method, unconfined compression test, granite rock

\section{Introduction}

Mesh convergence is a vital issue that must be addressed where it commonly affects the accuracy of the results analysed if overlooked. Thus, it is important to be comprehensively analysed in any given problem. Convergence is related to how small the elements needed to be to ensure that discrete element analysis results are not affected by changing the mesh size. Stress and deformation will usually converge to a repeatable solution with a reduction of the size of elements. It is necessary to demonstrate results converge to a solution and are not rely on mesh size. The proposed approach uses the DEM model to develop parameters for contact stiffness and failure theoretically. The convergence of elastic response and damage response due to crack growth is defined for the representative models considered in the analysis [1]. 
Jing \& Hudson [2] reviewed the application of numerical modelling for rock mechanics and rock engineering. The numerical methods most frequently used for rock mechanics problems can be categorised into three groups: continuum methods, discrete methods, and hybrid continuum/discrete methods. A mesh convergence studies were successful in replicating the rock experimental Unconfined Compression Test (UCT) based on element sizes using FEM [3] and FEM coupled with smooth particle hydrodynamics [4].

As crack surfaces are generated and evolved, fracturing and fragmentation are a challenge to numerical issues. Continuum methods solve partial differential equations of continuum mechanics, thus only considering a small number of discontinuities and cannot encompass the entire fracturing [5]. The difference between DEM and continuum-based methods is the contact patterns between system components constantly change for the former but are fixed for the latter with the deformation process [2]. Convergence mesh of DEM has been conducted, which subjected to compression loading to ensure the DEM model behaves like an actual rock [1], [6]-[8]. The combined finite-discrete element approach is used often to analyse the mechanism of fracturing rocks [9-11]. Munjiza \& John [12] investigated the effects of mesh sensitivity to the combined DEM-FEM fracture and fragmentation. They found out that there is a good indication of the required element size to obtain good results.

This paper aims to reproduce the results obtained from UCT experimental and investigate the effects of mesh element numbers by incorporating the DEM-FEM numerical simulation. Different types of mesh were conducted for UCT convergence mesh and compared with experimental tests. The comparison involved is the stress-strain curve trends, the failure behaviour, and the rock sample's compression strength.

\section{Discrete Element Method}

Cundall [13] suggested a rigid body dynamic particle simulation approach for fracturing cohesive frictional materials, common for geotechnical application. Cundall introduced the DEM for rock mechanics analyses and then used it on soils. Potyondy \& Cundall [14] stated that rock resembles a cemented granular material of complexly formed grains that deform and break grains and cement. Rock mechanical behaviour is influenced by the formation, growth, and existence of microcracks. The mechanical properties of the rock are determined by the constituent particles and structure [15].

Potyondy \& Cundall [14] defined Bonded Particle Model (BPM) as a dense particle packaging in either a circular or spherical non-uniform form. Each particle remains an independent particle, but the bonded particle model introduces bond at their contact points with a parallel bond. The bonded particle model can provide a research tool for studying micromechanisms and an engineering tool for predicting macroscopic behaviour. In BPM, the rigid particles are bound at the soft contact in normal and shear directions by parallel contacts with linear elastic springs. The illustration of a typical contact bond between particles is shown in Fig. 1, where the force and moment are acting at each contact. Bonds connect the bonded particles in BPM to the surrounding particles. Bonds reflect the entire mechanical behaviour, and bonds are DEM-independent. The strength of the micro shear is mobilised if the bonds are broken by shear or rotation. The shear strength is then set to residual strength, depending on the normal strength and friction coefficient, when contact has broken. In contrast, the bonding's tensile strength is set to zero regardless of its contact state if a tensile contact is broken [7].

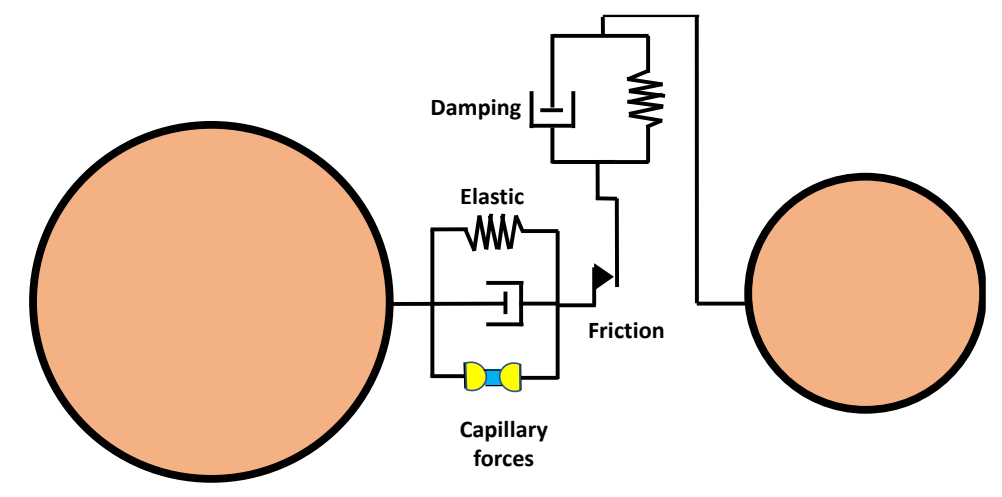

Fig. 1 - Illustration of the bond contact model between particles

Plesha [1] stated that a convergence analysis provides detail about how the interaction behaviour between the DEM elements is altered based on the element size. The convergence to the exact solution is achieved by reducing the DEM elements size during the refining process. This shows the important mesh convergence in DEM numerical simulation.

\section{Numerical Model}

Numerical simulation of the UCT was used for calibration purposes of the intact rock. The UCT numerical model was used to estimate the unconfined compressive strength of granite rock. The steps of calibrating the UCT intact rock 
were explained in [16]. Size of particles used in disc sphere generation, which is 2 to $2.5 \mathrm{~mm}$. Rock sample with $50 \mathrm{~mm}$ diameter and $100 \mathrm{~mm}$ height was used in the test. Fig. 2 shows the granite sample of an experimental and numerical model for the UCT test.

In the numerical model, two steel plates are positioned at the rock sample bottom and top. The top steel platen has been set moved vertically and fixed in all other directions, while all directions for the bottom steel platen are fixed. This replicates the actual laboratory test where only the top platen moves downward but is fixed at the bottom. Boundary condition with loading rate of $0.4 \mathrm{~mm} / \mathrm{min}$ was defined to the top steel platen, representing the actual experiment.

The steel platen material used in this study with a mass density of $7 \mathrm{~g} / \mathrm{cm}^{3}$, Young's modulus is equal to $200 \mathrm{GPa}$, and Poisson's ratio is 0.3 . Table 1 shows the assigned input rock properties for analysis in numerical simulation. The coefficient of friction between the steel plates and the rock specimen is 0.39 and 0.5 for the damping coefficient.

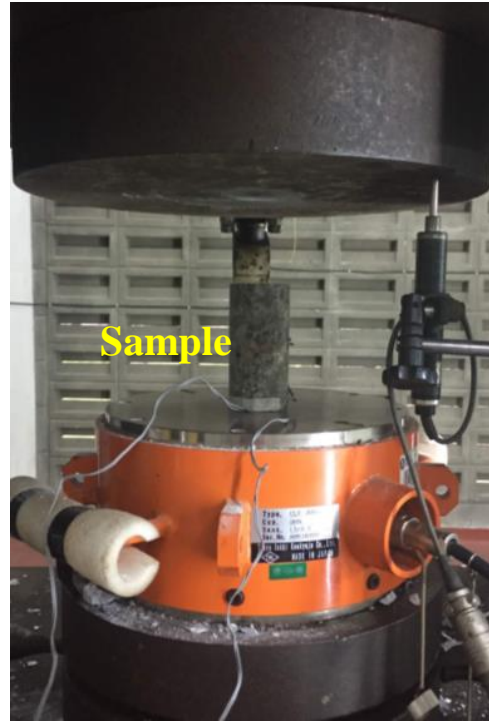

(a)

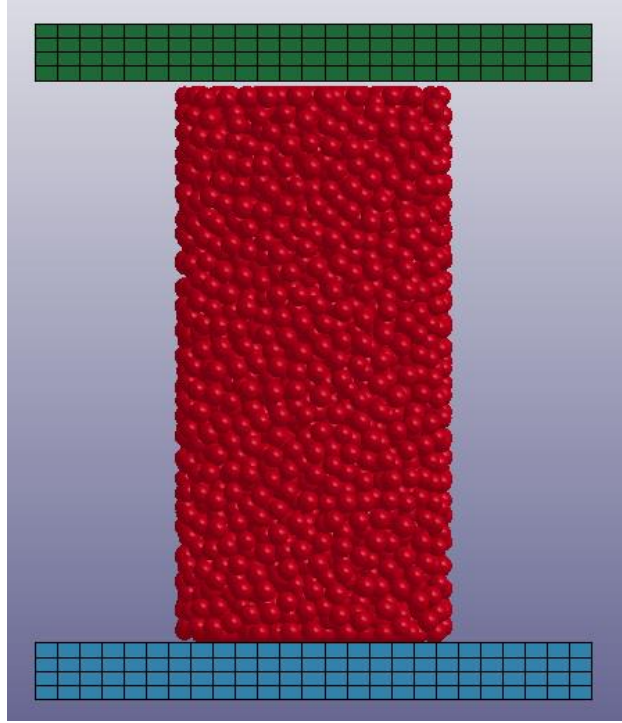

(b)

Fig. 2 - UCT of granite sample (a) experimental test; (b) numerical simulation

Table 1 - Rock properties used for numerical simulation [16]

\begin{tabular}{ccc}
\hline Test type & Parameter & Value \\
\hline Density test & Density $(\mathrm{g} / \mathrm{cm} 3)$ & 2.75 \\
& UCS $(\mathrm{MPa})$ & 87.96 \\
Unconfined Compression Test & E $(\mathrm{GPa})$ & 20.76 \\
& Poisson ratio & 0.25 \\
Brazilian test & Tensile strength $(\mathrm{MPa})$ & 9.57 \\
Shear test & Shear strength $(\mathrm{MPa})$ & 9.52 \\
\hline
\end{tabular}

This study has five different mesh types: normal mesh, fine mesh, super fine mesh, coarse mesh, and super coarse mesh. The mesh types are different from the number of elements in a circumferential direction and the number of elements along the cylinder's length. Table 2 below shows the "shape mesher" input parameters of different mesh types for the convergence effect. The illustrations of the different meshed models are shown in Fig. 3. The calibrated input micro-parameters of the UCT DEM model used in this study are shown in Table 3.

Table 2 - Input parameters of different mesh type for convergence effect

\begin{tabular}{cccccc}
\hline & Super Fine Mesh & Fine Mesh & Normal Mesh & Coarse Mesh & Super Coarse Mesh \\
\hline Radius (mm) & 25 & 25 & 25 & 25 & 25 \\
Length (mm) & 100 & 100 & 100 & 100 & 100 \\
No. element & 100 & 60 & 40 & 20 & 10 \\
No. & 75 & 45 & 30 & 15 & 7.5 \\
\hline
\end{tabular}

*No. element: Number of elements in circumferential direction

*No.: Number of elements along the length of the cylinder 

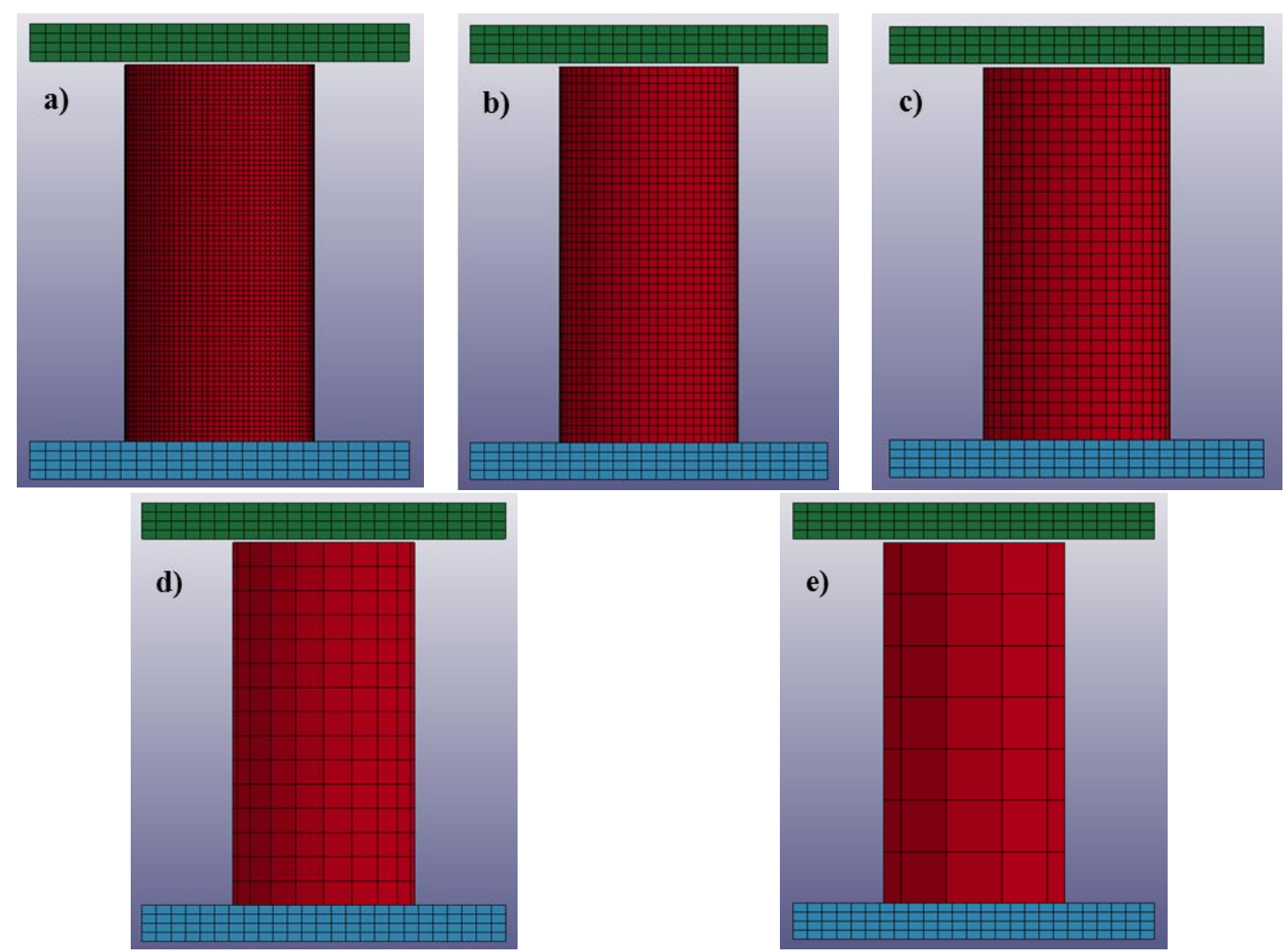

Fig. 3 - Comparison UCT DEM/BPM models mode of failure with different mesh types (a) Superfine mesh; (b) Fine mesh; (c) Normal mesh; (d) Coarse mesh; (e) Super coarse mesh

Table 3 - Input micro-properties of the UCT DEM/BPM model [16]

\begin{tabular}{|c|c|c|c|}
\hline Properties & Value & Properties & Value \\
\hline Particle radius / mm & $100-125$ & $\begin{array}{l}\text { Maximum gap between two bonded } \\
\text { spheres (MAXGAP) }\end{array}$ & -1.31 \\
\hline $\begin{array}{l}\text { Parallel-bond normal stiffness (PBN) / } \\
\text { GPa }\end{array}$ & 20.76 & Normal damping coefficient (NDAMP) & 0.70 \\
\hline Parallel-bond shear stiffness (PBS) & 0.25 & $\begin{array}{l}\text { Tangential damping coefficient } \\
\text { (TDAMP) }\end{array}$ & 0.01 \\
\hline $\begin{array}{l}\text { Parallel-bond maximum normal stress } \\
\text { (PBN_S) }\end{array}$ & 0.03 & Friction coefficient (Fric) & 0.99 \\
\hline $\begin{array}{l}\text { Parallel-bond maximum shear stress } \\
\text { (PBS_S) }\end{array}$ & 0.02 & Rolling friction coefficient (FricR) & 0.98 \\
\hline Bond radius multiplier (SFA) & 1.31 & Normal spring constant (NormK) & 0.10 \\
\hline Numerical damping (ALPHA) & 0.50 & Shear spring constant (ShearK) & 0.40 \\
\hline
\end{tabular}

\section{Results and Analysis}

Convergence effect was conducted to ensure that the discrete element analysis results are not affected by changing the mesh size. Five different mesh sizes were tested to determine the convergence effect. UC 1 to UC 5 represents the rock samples tested in the laboratory test. A comparison between axial compression stress and axial strain behaviour between DEM/BPM model and laboratory results is shown in Fig. 4. Good agreement was found where the similarity pattern was observed between the axial stress and strain of the DEM/BPM model with the experimental result of the UC 1 and UC 3.

Moreover, a comparison of uniaxial compressive strength also conducted, as shown in Fig. 5. It can be seen that the difference between the highest and lowest strength value in the convergence mesh is $17 \%$. The value of uniaxial compressive strength normal mesh and coarse mesh are the same, which shows it is converged and indicates no significant effect of mesh size in the models. 
Besides that, the failure behaviour of rock between the DEM/BPM model and experimental also was compared, as shown in Fig. 6. It can be seen that mesh types normal, fine, and superfine mesh failed in axial splitting, which is similar to the experimental test. In contrast, coarse and super coarse mesh types failed differently, which in shear modes of behaviour. Thus, the normal mesh was selected as the best mesh type in this study based on the comparisons that have been made.

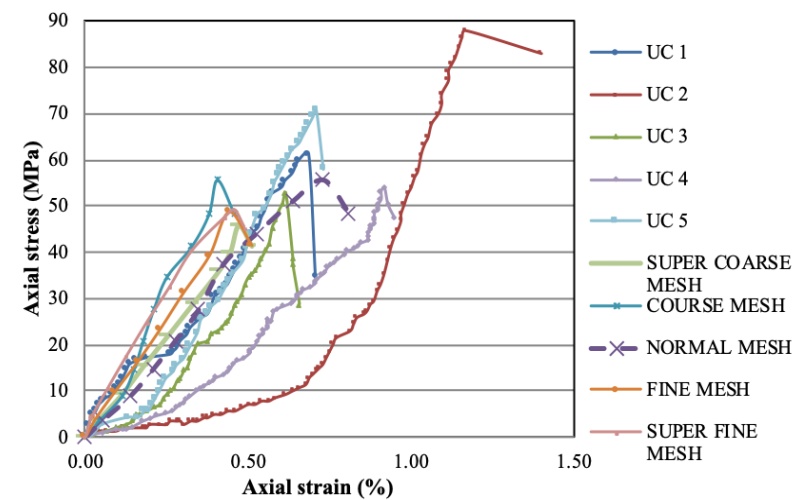

Fig. 4 - Comparison UCT DEM/BPM model with the different mesh size

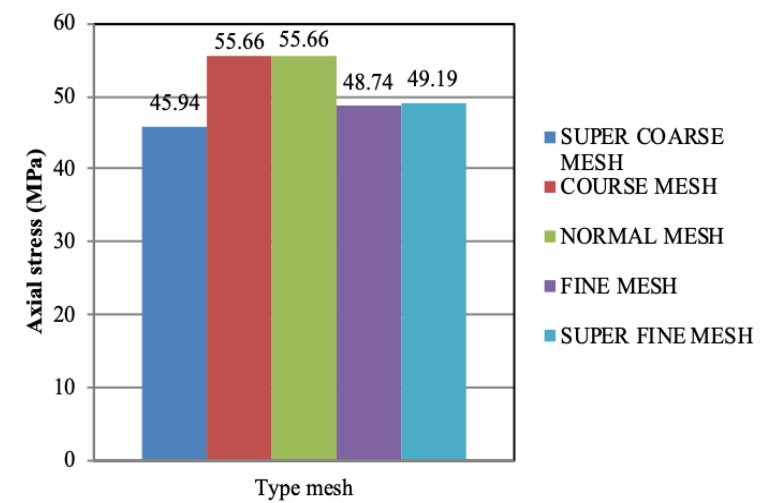

Fig. 5 - Comparison uniaxial compressive strength of different mesh UCT DEM/BPM model
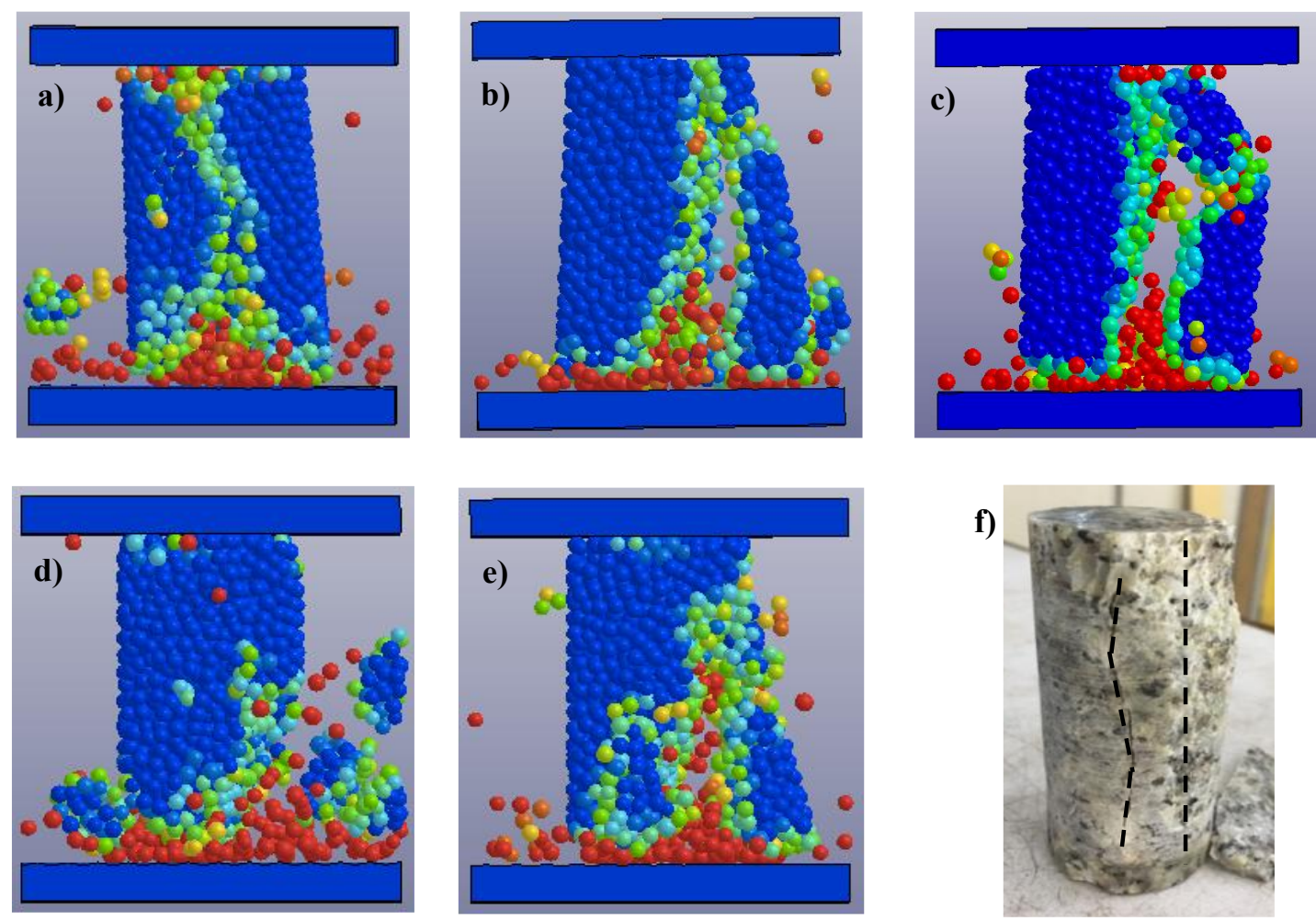

Fig. 6 - Models with different mesh types for convergence mesh a) Superfine mesh; b) Fine mesh; c) Normal mesh; d) Coarse mesh; e) Super coarse mesh; f) Experimental test

\section{Conclusion}

In this study, numerical simulation of DEM-FEM was used to investigate the effects of mesh element number on the UCT DEM-FEM model and the granite rock response under compression loading. The DEM-FEM numerical analysis was compared with the results obtained from the experimental test. It was found that the results are converged, and normal mesh is the best mesh type obtained in this study. The conducted numerical simulation can replicate the stress-strain curve trends, the failure behaviour, and fit the range of compression strength obtained in the experimental test. This shows that the DEM-FEM numerical simulation can represent the granite rock and be used for further study based on the mesh convergence results. 


\section{Acknowledgment}

The authors would like to thank the School of Civil Engineering, Faculty of Engineering, Universiti Teknologi Malaysia, Faculty of Engineering and Quantity Surveying, INTI International University, Nilai, Negeri Sembilan and Faculty of Civil Engineering, Universiti Teknologi MARA Pahang.

\section{References}

[1] Plesha M. E. (2003). Discrete Element Method for Modeling Penetration. Final Report No. 0704-0188, University of Wisconsin

[2] Jing L. \& Hudson J. A. (2002). Numerical methods in rock mechanics. International Journal of Rock Mechanics and Mining Sciences, 39, 409-27

[3] Mardalizad A., Manes A. \& Giglio M. (2016). An investigation in constitutive models for damage simulation of rock material. In AIAS - Associazione Italiana Per L'analisi Delle Sollecitazioni, Trieste

[4] Mardalizad A., Scazzosi R., Manes A. \& Giglio M. (2018). Testing and numerical simulation of a medium strength rock material under unconfined compression loading. Journal of Rock Mechanics and Geotechnical Engineering, 10, 197-211

[5] Carmona H. A., Wittel F. K. \& Kun F. (2014). From fracture to fragmentation: Discrete element modeling: Complexity of crackling noise and fragmentation phenomena revealed by discrete element simulations. European Physical Journal: Special Topics, 223, 2369-82

[6] Gibaud R. (2017) Application of the Discrete Element Method to Finite Inelastic Strain in Multi-Materials. PhD Thesis, Université Grenoble Alpes

[7] Li X. F., Zhang Q. B., Li H. B. \& Zhao J. (2018). Grain-based discrete element method (GB-DEM) modelling of multi-scale fracturing in rocks under dynamic loading. Rock Mechanics and Rock Engineering, 51(12), 37853817

[8] Madan N., Rojek J. \& Nosewicz S. (2019). Convergence and stability analysis of the deformable discrete element method. International Journal for Numerical Methods in Engineering, 118, 320-44

[9] Ma G., Zhang Y., Zhou W., Ng T. T., Wang Q. \& Chen X. (2018). The effect of different fracture mechanisms on impact fragmentation of brittle heterogeneous solid. International Journal of Impact Engineering, 113, 132-43

[10] Saksala T. (2016). Modelling of dynamic rock fracture process with a rate-dependent combined continuum damage-embedded discontinuity model incorporating microstructure. Rock Mechanics and Rock Engineering. 49(10), 3947-62

[11] Saksala T. (2015). Rate-dependent embedded discontinuity approach incorporating heterogeneity for numerical modeling of rock fracture. Rock Mechanics and Rock Engineering, 48, 1605-22

[12] Munjiza A. \& John N. W. M. (2002). Mesh size sensitivity of the combined FEM/DEM fracture and fragmentation algorithms. Engineering Fracture Mechanics, 69, 281-95

[13] Cundall P. A. \& Strack O. D. L. (1979). A discrete numerical model for granular assemblies. Géotechnique, 29, 47-65

[14] Potyondy D. O. \& Cundall P. A. (2004). A bonded-particle model for rock. International Journal of Rock Mechanics and Mining Sciences, 41, 1329-64

[15] Jaeger J. C., Cook N. G. W. \& Zimmerman R. W. (2007). Fundamentals of Rock Mechanics. Blackwell Publishing

[16] Shahrin M. I., Abdullah R. A., Jeon S. \& Sa'ari R. (2019). Calibration of rock uniaxial compression test using discrete element method in LS-DYNA. International Journal of Civil Engineering and Technology, 10, 975-84 